Journal of Case Reports 2017;7(3):289-292

\title{
BCR-ABL Positive Childhood Chronic Myeloid Leukemia
}

\author{
Timothy A. Ekwere ${ }^{1}$, Emmanuel K. Abudu ${ }^{2}$ \\ Department of ${ }^{1}$ Haematology and ${ }^{2}$ Pathology, College of Health Sciences, University of Uyo, Akwa Ibom State, Nigeria.
}

\begin{abstract}
Corresponding Author:
Dr. Timothy A. Ekwere

Email: timothyekwere@yahoo.com

This is an Open Access article distributed under the terms of the Creative Commons Attribution License (creativecommons.org/ licenses/by/3.0).
\end{abstract}

Received : April 3, 2017

Accepted : July 29, 2017

Published : August 15, 2017

\begin{abstract}
Background: Chronic myeloid leukemia (CML) is a rare disease entity in the pediatric age group constituting about $3-5 \%$ of childhood leukemia. The natural history and biologic characteristics of the disease are similar to adult CML. In addition, the response to therapy (tyrosine kinase inhibitor) is equally remarkable with no limiting side effect reported thus far. Case Report: We report a rare case of BCR-ABL positive CML in a 16 year old teenage girl. Conclusion: The aim of this study is to bring to the similarities in the molecular pathology and management between this very rare childhood leukemia and the adult-type.
\end{abstract}

Keywords: BCR-ABL Positive, Biological Products, Child, Leukemia.

\section{Introduction}

Chronic myeloid leukemia (CML) is a clonal hemopoietic neoplasm caused by acquired genetic abnormality in the pluripotent bone marrow stem cell, characterized by acquisition of the Philadelphia chromosome $(\mathrm{Ph})$ in the leukemic stem cells and its progeny [1]. The molecular biology of CML involves the reciprocal translocation of genes on chromosome 9 and 22. This results in the juxtaposition of the BCR gene on chromosome 22 with the virus $A B L$ gene. The resulting fusion protein $\mathrm{BCR}-\mathrm{ABL}$ has an enhanced tyrosine kinase activity resulting in enhanced myeloid proliferation and differentiation [2,3]. It is the first malignant disease to be associated with a genetic lesion and the first leukemia with genotype basis for molecular targeted therapy [3].

The occurrence of CML in children is quite rare. It constitute about $3-5 \%$ of all childhood leukemia with annual incidence of about 1 per million children [4]. However, the molecular biology of the disease in childhood is the same as adults; i.e. the balanced translocation $t(9: 22)$ that result in the fusion gene BCR-ABL [3]. We report a rare case of childhood CML in chronic phase.

\section{Case Report}

A 16 year old previously healthy girl presented to the Hematology clinic with a six weeks history of recurrent fever, progressive weakness and abdominal distension. The significant findings on clinical examination were severe pallor, and massive splenomegaly measuring approximately $20 \mathrm{~cm}$ below the left costal margin. She was afebrile with temperature of $36.8^{\circ} \mathrm{C}$ and weighed $48 \mathrm{~kg}$.

She was admitted and an urgent full blood count (FBC) showed: packed cell volume (PCV): 17\%; haematocrit: $0.17 \mathrm{~L} / \mathrm{L}$; leucocyte count (WBC): $261 \times 10^{9} / \mathrm{L}$; platelets $400 \times 10^{9} / \mathrm{L}$. Peripheral blood film (PBF) review revealed hyper-leucocytosis showing a complete spectrum of the myeloid cell series at different stages of maturation with peaks at myelocytes and metamyelocytes stages and basophila. Myelogram showed: myeloblast $02 \%$; promyelocyte $5 \%$; myelocyte $25 \%$; metamyelocyte $17 \%$; band forms $20 \%$; neutrophils $20 \%$; basophils $5 \%$, eosinophils $03 \%$, lymphocytes $03 \%$ [Fig 1,2].

The FBC and PBF results were suggestive of CML. The patient and care-giver (parents) were 
counselled on the course and prognosis of the disease. Initial supportive treatment was correction of anemia with two units of packed red cells, proper hydration with intravenous infusion of normal saline and oral xanthine oxidase inhibitor allopurinol to prevent tumour lysis syndrome. A cyto-reductive agent hydroxyurea (HU) was commenced at a dose of 1 gram daily.

Further evaluation was carried out to determine BCR-ABL transcript. The result was as follows: BCR-ABL 1 transcript type: e6a2 (using multiplex PCR method); ABL quantitation: $1.08 \times 10^{4}$ copies per $5 \mu \mathrm{L}$ of cDNA; BCR-ABL: quantity: 2390 copies per $5 \mu \mathrm{L}$ of cDNA (using Real Time PCR-TaqMan chemistry); BCR-ABL ratio: $22.13 \%$. With the $\mathrm{BCR}-\mathrm{ABL}$ transcript result, the diagnosis of adult-type CML was confirmed and patient was commenced on a tyrosine kinase inhibitor (TKI), Imatinib at a dose of $400 \mathrm{mg}$ daily in addition to the HU the dose of which was increased to 2 grams daily as a result of the hyper-leucocytosis. Following the commencement of the Imatinib, patient response was remarkable clinically as evident by progressive reduction and complete disappearance of the spleen, improvement in general sense of well-being and weight gain. A progressive improvement was observed in the hematological parameters and normal values of hemoglobin and total WBC count were attained within 8 weeks of commencing treatment [Table 1]. Currently, the patient is on routine clinic follow-up. Imatinib is well tolerated with no signs of adverse reaction reported thus far.

\section{Discussion}

Children and young adult constitute a small proportion of patients diagnosed with adult-type CML. This represents about $3-5 \%$ of all cases of newly diagnosed childhood leukemia [4]. The clinical course and prognosis are similar to that of adult CML. However, unlike the adult CML, ionizing radiation and other such environmental predisposing factors have not been shown to have a causal effect in its occurrence [4].

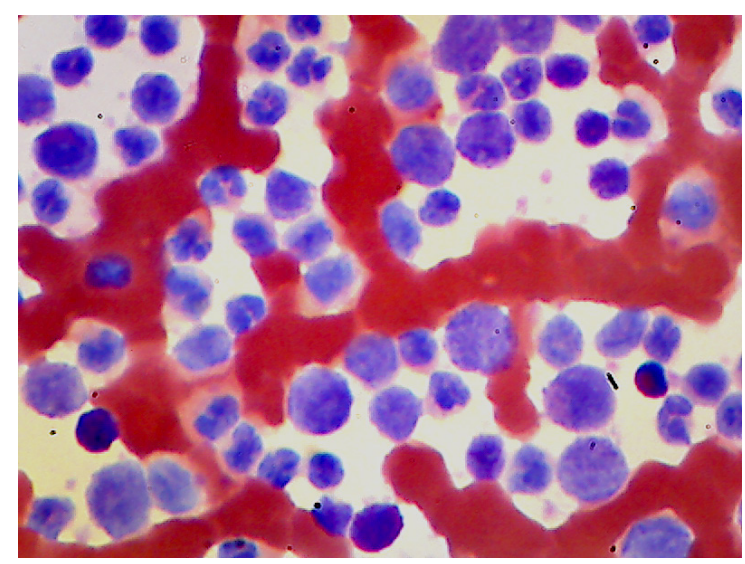

Fig 1: Peripheral blood film smear of patient showng hyperleucoytosis (Leishman stains x10).



Fig 2: Peripheral blood smear showing complete spectrum of the of myeloid cell series at different stages of maturation (Leishman stain $\times 40$ ).

The natural history of childhood leukemia is similar to that of the adult-type; progressing through the chronic phase characterized by expansion of the myeloid cell series in the bone marrow (BM) and defined by less than $10 \%$ blasts in the BM. Over 95\% children presents in chronic phase classically with features of anemia, leucocytosis and thrombocytosis [5]. Our index patient presented in this phase with a relatively high WBC counts. Studies by previous authors have shown that children generally presents with a higher WBC count compared to adults [5]. Millot et al. [5] reported a median WBC count of $242 \times 10^{9} / \mathrm{L}$ in children compared to median WBC count of $12-174 \times 10^{9} / \mathrm{L}$ in adults. 
Table 1: Haematological Profile of patient with Medication.

\begin{tabular}{|l|l|l|l|l|l|l|}
\hline S.No. & Day of Admission & Hb. g/dL & PCV $\%$ & WBC $\times / \mathbf{L}$ & Platelets/L & Medication \\
\hline 1 & 1 & 7.00 & 17 & $261 \times 10^{9}$ & $400 \times 10^{9}$ & HU \\
\hline 2 & 4 & 8.20 & 23 & $259 \times 10^{9}$ & $335 \times 10^{9}$ & HU \\
\hline 3 & 11 & 8.20 & 23 & $185 \times 10^{9}$ & $277 \times 10^{9}$ & TKI+HU \\
\hline 4 & 18 & 8.60 & 27 & $162 \times 10^{9}$ & $447 \times 10^{9}$ & TKI + HU \\
\hline 5 & 37 & 9.00 & 28 & $144.5 \times 10^{9}$ & $451 \times 10^{9}$ & TKI + HU \\
\hline 6 & 66 & 8.20 & 26 & $32.7 \times 10^{9}$ & $213 \times 10^{9}$ & TKI + HU \\
\hline 7 & 75 & 11.00 & 34 & $5.1 \times 10^{9}$ & $315 \times 10^{9}$ & TKI \\
\hline 8 & 128 & 11.00 & 33 & $5.4 \times 10^{9}$ & $583 \times 10^{9}$ & TKI \\
\hline
\end{tabular}

Hb: hemoglobin; PCV: packed cell volume; WBC: white blood cells; HU: hydroxyurea; TKI: tyrosine kinase inhibitor.

The accelerated phase is defined by the presence of BM blasts of between 10-19\%, peripheral blood basophilia of more than $20 \%$, persistent thrombocytosis $\left(>1000 \times 10^{9} / \mathrm{L}\right)$ or thrombocytopenia $\left(<100 \times 10^{9} / \mathrm{L}\right)$, increasing splenic size and evolution of new cytogenetic abnormalities. This phase is less common in children. The third phase otherwise called the blast crisis presents as overt acute leukemia, commonly acute myeloid leukemia (AML) and is defined by more than $20 \%$ blasts in the BM or extra-medullary proliferation of blasts [6].

The diagnosis of CML has evolved over time, beginning from the conventional cytogenetic analysis involving the culturing of BM sample to detect or demonstrate the presence $\left(\mathrm{Ph}^{+}\right)$or absence $\left(\mathrm{Ph}^{-}\right)$of the Philadelphia $(\mathrm{Ph})$ chromosome defined by the reciprocal translocation between chromosome 9 and 22 [7]. The fluorescence insitu hybridization (FISH) is a more advanced technique for chromosomal analysis. FISH can be used to determine specific region on the BCR-ABL gene using either blood or BM samples without necessarily culturing the cells [7]. The polymerase chain reaction (PCR) is super-sensitive advanced molecular technique that can be used to determine BCR-ABL oncogene in leukemic cell using either peripheral blood or BM samples. This technique is able to detect very minute quantities of BCRABL transcript even when $\mathrm{Ph}$ cannot be detected by conventional cytogenetic procedures. The sensitivity of this procedure makes it invaluable in assessing minimal residual disease especially in the monitoring of $\mathrm{BCR}-\mathrm{ABL}$ transcripts in $\mathrm{CML}$ patients [7].

The confirmation of CML in our index patient was based on PCR technique. For us the clinical significance of using this technique was to explore the option of using molecular targeted therapy as treatment modality given that the potential curative treatment hemopoietic stem cell transplantation (HSCT) is presently not available in our centre. The use of this molecular diagnostic technique (PCR) is now considered an essential part of modern management of CML patients especially in patients whose bone marrow karyotype did not demonstrate the $\mathrm{Ph}$ chromosome [8].

The introduction of TKI a molecular targeted therapy revolutionized the treatment of adult CML [9]. However, data on it use in pediatric age group is much limited. A review of the French National phase 4 clinical trial revealed that of the 44 children with CML in chronic phase enrolled in the study, $98 \%$ of the children achieved complete hematological response (CHR) while 61\% and $31 \%$ achieved complete cytogenetic (CCyR) and major molecular response (MMR) respectively in the first year of treatment with Imatinib $260 \mathrm{mg} / \mathrm{m}^{2}$ [10]. Similarly, Kobel et al. [11], reported CHR, CCyR and complete molecular response (CMR) in each of 5 children with $\mathrm{Ph}^{+}$leukaemia who were 
on Imatinb at a median period of 30, 285 and 287 days respectively. No limiting side effect was reported in any of the children during the study period. Based on the impressive results of the few available clinical trials, Imatinib is now being implemented in the primary treatment protocol for childhood CML. However, its potential to achieve a permanent cure is still in doubt given the paucity of data on it long term use with attendant possible side effects that may arise from its prolonged use in children.

Our index patient was commenced on Imatinib at a dose of $400 \mathrm{mg}$ daily initially in combination with HU because of the high WBC count. CHR and clinical response was observed within 8-weeks (approximately 60 days) after the commencement of treatment. This was in keeping with findings from other similar reports [11]. However, molecular and cytogenetic response could not be assessed because of limited financial resources.

\section{Conclusion}

Childhood CML is a relatively rare disease entity that shares the same molecular and biologic characteristics with adult type CML. Treatment with TKI is quite favourable though there is still paucity of data on long time use in pediatric age group with respect to permanent cure.

Contributors: TAE: acquisition and review of patient's medical records including laboratory investigations of current disease, review of patient's slides, drafting the article; EKA: review of patient's slides, revising it critically for important intellectual content. TAE will act as guarantor. Both authors approved the final version of the manuscript.

Funding: None; Competing interests: None stated.

\section{References}

1. Ibegbulsm O, Ezekwesili A, Okpala I. Leukaemia in Adults. In: Okpala I, Johnson C, editors. Synopsis of Haematology including blood diseases in the tropics. $1^{\text {st }}$ ed. 2010. pp.111-115.

2. Cortes J, Deininger M. BCR-ABL as a molecular target. In: Deininger M, editor. Chronic Myeloid Leukemia. $1^{\text {st }}$ ed. New York: Informa Healthcare; 2006. pp. 27-45.

3. Sawyers CL. Chronic myeloid leukaemia. N Engl J Med. 1999;340:1330-1340.

4. Andolina JR, Neudorf SM, Corey SJ. How I treat childhood CML. Blood. 2012;119:1821-1830.

5. Millot F, Traore P, Guilhot J, Nelken B, Leblanc T, Leverger $\mathrm{G}$, et al. Clinical and biological features at diagnosis in 40 children with chronic myeloid leukemia. Paediatrics. 2005;116:140-143.

6. Vardiman JW, Melo JV, Baccarani M, Thiele J. Chronic myelogenous leukemia, BCR-ABL1 positive. In: Swerdlow SH, Campo E, Harris NL, Jaffe ES, Pileri SA, Stein $\mathrm{H}$, et al, editors. WHO classification of tumours of hematopoietic and lymphoid tissues. Lyon: IARC; 2008. pp. 32-37.

7. Lange T, Deininger MW. Molecular diagnostics in chronic myeloid leukaemia. Expert Opin Med Diagn. 2010;4:113-124.

8. Crampe M, Haslam K, Kelly J, Conneally E, Langabeer $\mathrm{S}$. Characterization of a novel variant BCR-ABL1 fusion transcript in a patient with chronic myeloid leukemia: Implications for molecular monitoring. Hematol Oncol and Stem Cell Ther. 2016;S 1858-3876:30002-30004

9. O'Brien SG, Guilhot F, Larson RA, Gathmann L, Baccarani M, Cervantes F, et al. Imatinib compared with interferon and low-dose cytarabine for newly diagnosed chronic phase chronic myeloid leukemia. N Engl J Med. 2003;348:994-1004.

10. Millot F, Guilhot J, Nelken B, Leblanc T, De Bont ES, Bekassy AN, et al. Imatinib mesylate is effective in children with chronic myelogenous leukemia in late chronic and advanced phase and in relapse after stem cell transplantation. Leukaemia. 2006;20:187-192.

11. Kolb EA, Pan Q, Ladanyi M, Steinherz PG. Imatinib mesylate in Philadelphia chromosome-positive leukemia of childhood. Cancer. 2003;98:2643-2650. 